\title{
Author Correction: Glis1 facilitates induction of pluripotency via an epigenome- metabolome-epigenome signalling cascade
}

Linpeng Li(D), Keshi Chen (D), Tianyu Wang (iD, Yi Wu (D), Guangsuo Xing (D), Mengqi Chen (D), Zhihong Hao (iD, Cheng Zhang (D), Jinye Zhang (D), Bochao Ma (D), Zihuang Liu(D), Hao Yuan (D), Zijian Liu (D), Qi Long (D), Yanshuang Zhou (D), Juntao Qi DiD, Danyun Zhao (D), Mi Gao DiD, Duanqing Pei, Jinfu Nie, Dan Ye, Guangjin Pan (D) and Xingguo Liu (D)

Correction to: Nature Metabolism https://doi.org/10.1038/s42255-020-0267-9, published online 24 August 2020.

In the version of this article initially published, in the graph key in Fig. 3a, the labels for 'SKO + Flag' and 'SKO + Glis1' were switched; 'SKO + Flag' should be blue, and 'SKO + Glis1' should be red. In Extended Data Fig. 1c, the $\beta$-gal image of P7-MEFs mistakenly duplicated that of P8-Flag MEFs. The errors have been corrected in the HTML and PDF versions of the article.

C

Original

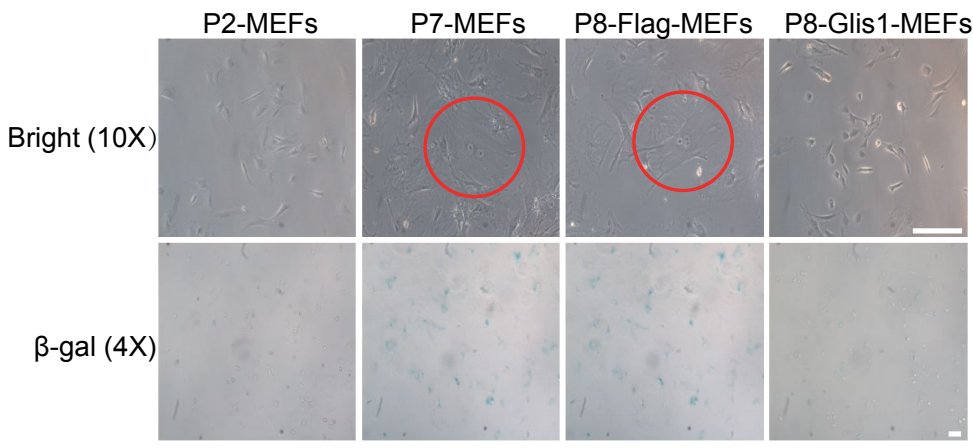

C

Corrected

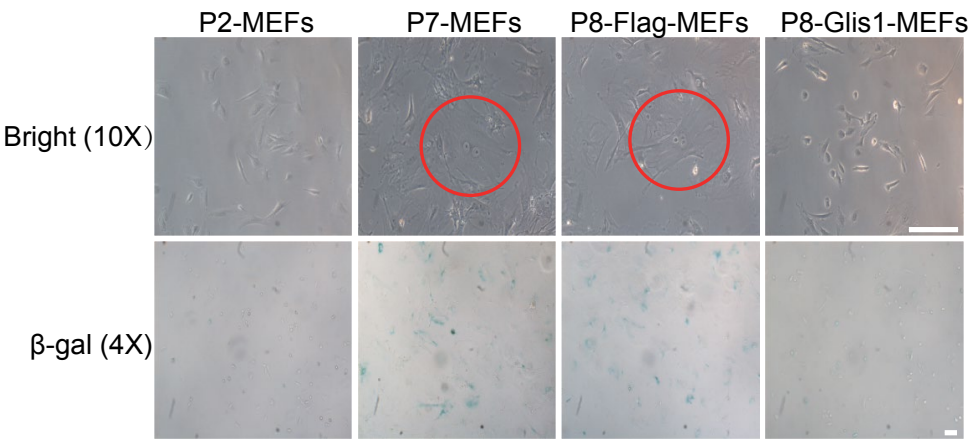

Extended Data Fig. 1 | Original and corrected.

Published online: 8 October 2020

https://doi.org/10.1038/s42255-020-00308-0

(C) The Author(s), under exclusive licence to Springer Nature Limited 2020 